Ann. Biol. anim. Bioch. Biophys., I973, 13 (2), 285-305.

REVUE BIBLIOGRAPHIQUE

\title{
ESTIMATION DE LA COMPOSITION CORPORELLE DES ANIMAUX \\ A PARTIR DES ESPACES DE DIFFUSION DE L'EAU MARQUÉE
}

\author{
J. ROBELIN \\ Station de Recherches sur l'Élevage des Ruminants, \\ Centre de Recherches zootechniques et vétérinaires, $I$. N. R. A., \\ Theix 63110 Beaumont
}

\section{RÉSUMÉ}

Parmi les méthodes indirectes d'estimation de la composition corporelle sur l'animal vivant, la méthode fondée sur la dilution d'un marqueur de l'eau semble la plus prometteuse.

D'après les nombreux travaux effectués depuis bientôt 40 années sur ce sujet, il semble que l'eau marquée (eau lourde ou eau tritiée) donne les meilleurs résultats.

La diffusion dans l'eau corporelle est rapide, I à 7 heures suivant les espèces, elle est homogène dans les différents compartiments hydriques de l'organisme, l'élimination du marqueur est lente (I p. Ioo par heure de la dose contenue dans l'organisme).

La liaison entre l'espace de diffusion de l'eau marquée et le volume hydrique réel est forte $(r>0,96)$; elle est plus faible entre cet espace de diffusion rapporté au poids vif, et la teneur en matières grasses de l'animal $(r=-0,92$ à $-0,99)$.

La précision de cette méthode d'estimation est limitée, surtout dans le cas des ruminants, par l'importance et la variabilité de la quantité d'eau contenue dans le tube digestif.

La connaissance de la composition corporelle et plus encore de ses variations présente un intérêt majeur dans de nombreux secteurs de la biologie : physiologie de la croissance et du développement, nutrition, génétique... Elle permet en effet, d'apprécier avec plus de finesse que la pesée seule, un ensemble aussi hétérogène que le corps d'un animal. Cette connaissance peut également être fondamentale pour le pathologiste, notamment en clinique humaine, dans l'étude des déséquilibres métaboliques.

Annales de Biologie animale. - r 973. 
En ce qui concerne le cas des animaux domestiques, la méthode la plus directe pour évaluer la composition corporelle est l'abattage suivi de la dissection et de l'analyse du corps entier. Pour des raisons de facilité, on limite souvent l'étude à la partie commercialisable, c'est-à-dire la carcasse. Ce procédé n'en demeure pas moins long et coûteux ; c'est pourquoi on a tenté de le remplacer, dans le cas des bovins, par une estimation indirecte à partir de la composition anatomique ou chimique d'éléments supposés représentatifs de la carcasse (HOPPER, I944; Fiankins et Howe, 1946; Crown et Damon, I960; Ledger et Hutchison, I962; Martin et Torreele, i967 : Geay et Beranger, I969) (tabl. I) ou à partir de la densité de la carcasse elle-même (Rathbun et Pace, 1945 ; Kraybill et al., 1952 ; Garrett et Hinman, 1969 ; Desmoulin, 1970).

\section{TABLEAU I}

Morceaux utilisés pour l'estimation indirecte de la composition des carcasses de bovins

\begin{tabular}{|c|c|c|}
\hline Auteurs & Morceaux utilisés & $R\left({ }^{(1)}\right.$ \\
\hline 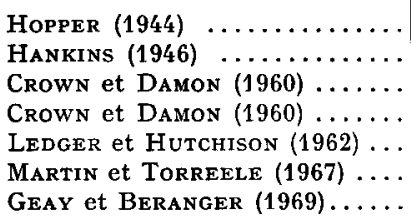 & $\begin{array}{c}\text { 9-10-11 e côtes } \\
9-10-11 \text { e côtes } \\
12^{\mathrm{e}} \text { côte } \\
9-10-11^{\mathrm{e}} \text { côtes } \\
10^{\mathrm{e}} \text { côte } \\
7-8-9^{\mathrm{e}} \text { côtes } \\
11^{\mathrm{e}} \text { côte }\end{array}$ & $\begin{array}{l}0,984 \\
0,93 \\
0,962 \\
0,976 \\
0,963 \\
0,974 \\
0,948\end{array}$ \\
\hline
\end{tabular}

(1) R : Coefficient de corrélation entre la teneur en dépôts adipeux dans le morceau et dans la carcasse.

Ces méthodes, directes ou indirectes, d'estimation post mortem de la composition de la carcasse ou du corps entier, présentent l'inconvénient de rendre très difficiles les études cinétiques du développement, puisque dans ce cas, il est nécessaire d'analyser successivement à des âges différents, des animaux supposés être comparables dans leur évolution corporelle. Il ne faut pas négliger non plus le sacrifice financier énorme que cela nécessite, surtout dans le cas des gros ruminants.

C'est pourquoi, depuis 30 ans déjà, de nombreux auteurs ont cherché à évaluer la composition corporelle sur l'animal vivant.

Les diverses méthodes proposées ont fait l'objet de revues bibliographiques détaillées (SirI, I956; Dumont, I958; Brozeck, 1963; Panaretto, r968) : mesure de l'épaisseur des dépôts adipeux sous-cutanés par ultra-sons, densité sur l'animal vivant, diffusion de gaz solubles dans les lipides, espaces de diffusion... Il en ressort que l'estimation de la composition corporelle à partir du volume hydrique, déterminé par diffusion d'un marqueur de l'eau, présente de nombreux avantages par rapport aux autres méthodes, tant en précision qu'en facilité de mise en œuvre.

Différents marqueurs ont été utilisés dans ce but (urée, thiourée, sulfanilamide, antipyrine, $\mathrm{N}$-acétylaminoantipyrine, eau lourde, eau tritiée) (tabl. 2). D'après Dumont (1958) et Panaretto et Till (I963. II), HAXHE (I964) et KAy (I966), il apparaît que l'eau marquée (eau lourde où eau tritiée) permet d'obtenir les résultats les plus précis.

Cette revue bibliographique a donc pour objectif de faire une mise au point des connaissances actuelles relatives à l'utilisation de l'eau marquée, afin de préciser les conditions de sa mise en œuvre et ses limites d'emploi. 


\section{TABLEAU 2}

Marqueurs utilisés pour déterminer la teneur en eau sur des animaux vivants

\begin{tabular}{|c|c|}
\hline Auteurs & Marqueurs \\
\hline $\begin{array}{l}\text { Hevesy (1934) } \\
\text { Painter (1940) } \\
\text { Painter (1940) } \\
\text { Danowski (1944) } \\
\text { Pace (1947) } \\
\text { Soberman (1949) } \\
\text { Brodie (1951) }\end{array}$ & $\begin{array}{l}\text { Eau lourde } \\
\text { Sulfanilamide } \\
\text { Urée } \\
\text { Thiourée } \\
\text { Eau tritiée } \\
\text { Antipyrine } \\
\text { N-aminoacétylantipyrine }\end{array}$ \\
\hline
\end{tabular}

Après une analyse théorique du principe de la méthode, nous étudierons le devenir de l'eau marquée dans l'organisme, pour examiner ensuite de façon critique les résultats obtenus au niveau de l'estimation de la composition corporelle.

\section{I. - PRINCIPES DE LA MÉTHODE}

Cette méthode d'estimation de la composition corporelle repose sur deux principes, à savoir : I $^{\circ}$ A partir d'un certain stade de développement, la composition corporelle est liée au volume hydrique de l'organisme.

$2^{0}$ Le volume hydrique de l'organisme est lié à l'espace de diffusion de l'eau marquée.

Io Le premier principe s'appuie sur les observations de Murray (1922) et Moulton et al. (I922) qui montrent que la composition chimique de la masse délipidée des animaux est pratiquement constante ( $73 \mathrm{p}$. I0o d'eau, $22 \mathrm{p}$. Ioo de protéines et $5 \mathrm{p}$. 100 de matières minérales environ). Moulton (1923) confirme ces résultats en précisant qu'ils ne sont valables qu'à partir d'un certain stade physiologique, "maturité chimique", qui se situerait à un âge voisin de 5 mois chez les bovins. De nombreux auteurs (cf. PACE et al., I945), ont ensuite vérifié ces constatations sur différentes espèces (Rat, Cobaye, Lapin, Chat, Chien, Singe). Ainsi, connaissant la teneur en eau de la masse délipidée, le poids vif, et le volume hydrique d'un animal, on peut calculer sa teneur en lipides.

$$
\text { lipides p. Ioo poids vif }=\text { I00 }-\left(\frac{\text { eau p. roo poids vif }}{\text { eau p. Ioo masse délipidée }}\right)
$$

En réalité, la composition chimique de la masse délipidée n'est pas rigoureusement constante avec l'âge, comme l'ont montré REID et al, (I955). Aussi est-il plus judicieux de rechercher une relation statistique directe entre la teneur en eau et la teneur en lipides de l'organisme.

Le tableau 3 présente quelques données bibliographiques relatives à cette relation, dans le cas de différentes espèces animales. La liaison est très étroite $(r$ voisin de $-0,99)$ et l'erreur résiduelle relativement faible (I,5 $\mathrm{p}$. Ioo du poids vif vide).

Comme l'ont montré Reid (I955), Reid et al. (I968) et Garrett et Hinman (I969), on peut également établir une liaison statistique entre les teneurs en eau et en matières grasses obtenues 
à partir des résultats précédents et la teneur en azote. Le coefficient de corrélation est égal o,99, et l'erreur résiduelle voisine de o, I p. Ioo pour des animaux dont la teneur en azote est d'environ 2,7 p. Ioo du poids vif vide (poids vif diminué du contenu digestif).

\section{TABLEAU 3}

Relation entre la teneur en eau et la teneur en lipides de l'organisme

\begin{tabular}{|c|c|c|c|c|c|}
\hline Auteurs & Animaux & $\begin{array}{c}\text { Nombre } \\
\text { d'animaux }\end{array}$ & $\begin{array}{c}\text { Teneur } \\
\text { en lipides }\end{array}$ & $\mathrm{R}_{y x}$ & $\mathrm{~S}_{y x}$ \\
\hline 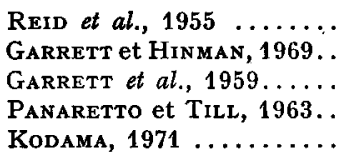 & $\begin{array}{l}\text { bovins } \\
\text { bovins } \\
\text { ovins } \\
\text { caprins } \\
\text { hamsters }\end{array}$ & $\begin{array}{r}256 \\
48 \\
59 \\
13 \\
34\end{array}$ & $\begin{aligned} 1,8 & \text { à } 44,6 \\
15,0 & \text { à } 35,0 \\
& \\
0,6 & \text { à } 26,7 \\
10 & \text { à } 30\end{aligned}$ & $\begin{array}{l}-0,987 \\
-0,99 \\
-0,989 \\
-0,982 \\
-0,98\end{array}$ & $\begin{array}{l}1,440 \\
0,82 \\
1,5 \\
0,81\end{array}$ \\
\hline
\end{tabular}

$y=$ Lipides p. $100 \mathrm{du}$ poids vif.

$x=$ Eau p. 100 du poids vif.

$\mathrm{R}_{y x}=$ Coefficient de corrélation entre $y$ et $x$.

$\mathrm{S}_{y x}=$ Écart résiduel de la régression de $y$ sur $x$.

En admettant le premier principe, on peut estimer de façon relativement précise la composition chimique d'un animal, en ne connaissant que son poids vif et son volume hydrique.

$2^{\circ}$ Le deuxième principe sur lequel repose la méthode, suppose qu'on peut assimiler le volume hydrique corporel à l'espace de diffusion d'un marqueur de l'eau, espace de diffusion qui est, selon HambURGER et Mathe (I952), "le volume hydrique apparent dans lequel cette substance s'est répandue de façon homogène lorsque sa diffusion est complète ".

Afin de voir par quel moyen on peut estimer ce volume, il paraît souhaitable de tracer schématiquement le devenir d'un marqueur idéal après son introduction dans l'organisme (fig. $\mathrm{r}$ ).

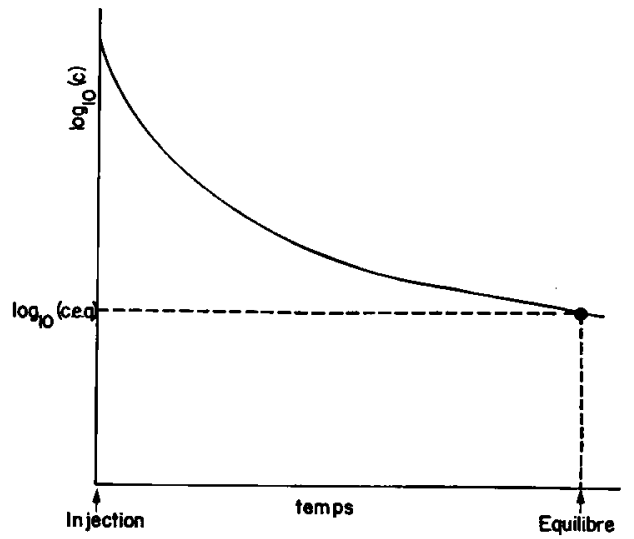

FIG. r. - Schéma de l'évolution en fonction du temps de la concentration d'un marqueur dans l'eau corporelle (d'après EDELman, I952)

$c=$ concentration du marqueur à l'instant $t$

ceq $=$ concentration au moment de l'équilibre (théorique) 
Lorsqu'on suit l'évolution de la concentration du marqueur dans l'eau plasmatique après son injection par voie intraveineuse, on observe une diminution rapide correspondant à la diffusion du marqueur. Cette phase conduit à un état d'équilibre. Ensuite, la décroissance devient plus lente et se traduit graphiquement, en coordonnées semi-logarithmiques, par une droite correspondant à l'élimination de la substance (Pinson et Langham, I957). Évidemment, cette description est schématique car l'élimination se manifeste dès l'introduction du marqueur dans l'organisme, mais ses effets sont masqués par la diffusion.

Le volume apparent occupé par le marqueur est égal à $\mathrm{Q} / \mathrm{C}, \mathrm{Q}$ étant la quantité de marqueur présente dans l'organisme, $\mathrm{C}$ sa concentration dans l'eau plasmatique, reflet de la concentration théorique dans l'eau corporelle totale. Dans la pratique, on peut.mesurer Qo, la quantité injectée, et $\mathrm{C}_{e q}$, la concentration à l'équilibre, mais on ne connaît, ni $Q_{e q}$, quantité présente à l'équilibre, ni $\mathrm{C}_{0}$, concentration théorique initiale. Le calcul du volume apparent ne peut donc se faire qu'à partir de l'estimation de l'une de ces deux valeurs.

On peut estimer $Q_{e q}$ en retranchant de $Q_{0}$ les pertes de marqueur, par l'urine en supposant que l'elimination ne se fasse que par l'urine et qu'il n'y ait pas de transformation du marqueur.

On peut estimer également $\mathrm{Co}$, en extrapolant la droite d'élimination au temps correspondant à l'injection (CACHERA et BARBIER, I94I). On suppose alors que si la diffusion avait été immédiate, l'élimination aurait été identique.

La détermination du volume hydrique d'un animal par la dilution d'un marqueur dans l'eau corporelle, suppose donc que cette substance réponde à un certain nombre de conditions :

- elle ne doit pas être toxique,

- elle doit diffuser rapidement et de façon homogène dans toute l'eau corporelle,

- elle ne doit pas diffuser à l'extérieur des compartiments hydriques de l'organisme,

- elle doit être éliminée suffisamment lentement pour que sa concentration soit encore appréciable dans l'eau plasmatique après équilibre.

Ces conditions étant définies, nous pouvons analyser la dilution de l'eau marquée, a fin d'estimer d'un point de vue théorique, ses possibilités d'application à la mesure de l'eau totale sur l'animal vivant.

\section{II. — ÉTUDE THÉORIQUE DE I,A DILUTION DE L'EAU MARQUÉE (EAU LOURDE, EAU TRITIÉE) DANS L'EAU CORPORELLE}

Nous nous proposons d'analyser simultanément la dilution de l'eau lourde (DOH) et de l'eau tritiée (TOH), leurs propriétés physico-chimiques étant relativement voisines en ce qui concerne les phénomènes de diffusion (Prentice et al., I952; Grascock et Duncombe, I954; Bowen, I960).

L'eau deutériée n'est pas toxique et l'injection d'eau tritiée ne semble pas poser de problème à des doses assez élevées $(0,2 \mathrm{mCi}$ par $100 \mathrm{~kg}$ de poids vif), pour permettre une mesure facile dans l'eau plasmatique (Hevezy et Hofer, 1934 ; Prentice et al., I952 ; Pinson et al., 1952-I957 ; Thompson, I954 Done et Payne, I957). L'effet isotopique de ces substances peut être négligé, compte tenu de la faible concentration utilisée (HEvesy et JACoBSEN, I940; GLASCOcK et DuNCOMBE, 1954 ; Dumonr, 1958). Notre étude portera donc essentiellement sur la diffusion du marqueur dans l'organisme, et sur son élimination ultérieure.

\section{A. - Diffusion}

La distribution du marqueur dans l'organisme se fait théoriquement, d'après les travaux de Hevesy et JACOBSEN (I940) en trois étapes. Les molécules diffusent tout d'abord dans l'eau plasmatique, traversent la paroi vasculaire pour occuper tout l'espace extracellulaire, puis occupent finalement l'espace intracellulaire. 
Dans la pratique, Enelman (1952) et Pinson (1952) ne décomposent la diffusion qu'en deux phases seulement; l'évolution de la concentration du marqueur dans l'eau plasmatique suit une loi de la forme

$$
\mathrm{C}_{t}=a_{1} \mathrm{e}^{-k_{1} t}+a_{2} \mathrm{e}^{-k_{2} t}+\mathrm{C}_{e q} \quad \text { (fig. I) }
$$

$\mathrm{C}^{t}=$ concentration du marqueur dans l'eau plasmatique à l'instant $t$

$\mathrm{C}_{e q}=$ concentration du marqueur dans l'eau plasmatique après diffusion complète

$a_{1}, a_{2}=$ constantes

$k_{1}, k_{2}=$ vitesses de diffusion

$k_{1}$ et $k_{2}$ correspondent à deux compartiments hydriques différents, dans lesquels la dilution du marqueur se fait à deux vitesses différentes. Chez le Chat et le Chien ainsi que l'Homme, la quantité de marqueur diffusant par minute, exprimée en pourcentage de la dose injectée, est voisine de 1,5 pour $k_{1}$ et 0,2 pour $k_{2}$. Ainsi, on atteint théoriquement l'équilibre à 99 p. Ioo dans ces compartiments en 20 et $60 \mathrm{mn}$ respectivement.

La question se pose de savoir à quels compartiments physiologiques correspondent ces deux vitesses. D'après les travaux de FleXNer et al. (1942) sur le cobaye, Hevesy et JACobsen (I940) sur le Lapin et Edelman (1952) sur le Chat, le Chien et l'Homme, il semble qu'aucun de ces compartiments ne puisse se superposer à l'espace extracellulaire. En effet, le passage de l'eau marquée à travers les vaisseaux sanguins est très rapide (FLEXNER, I942), et l'occupation de l'espace extracellulaire en entier ne demande pas plus d'une minute in vivo chez le Chien et in vitro sur le muscle en perfusion. Les deux compartiments appartiendraient donc à l'espace intracellulaire à l'intérieur duquel la diffusion est plus lente. En outre, EDELMAN (I952) montre que l'équilibre complet du marqueur n'est pas atteint dans le même temps dans les différents tissus ou organes du chien : $2 \mathrm{mn}$ dans le cerveau, Io à $20 \mathrm{mn}$ dans le foie, 2 à 3 heures dans le muscle et l'os. La résultante des différents compartiments de ces organes pourrait peut-être correspondre aux deux compartiments mis en évidence précédemment.

Ainsi, la diffusion du marqueur est très rapide dans l'espace extracellulaire ; elle est beaucoup plus lente et se produit en deux étapes dans l'eau intracellulaire. Ces conclusions ne sont pas en accord avec celles de Pinson (1952) qui pense que l'une des barrières s'opposant à la diffusion du marqueur est le cheminement des molécules d'eau marquée dans les capillaires sanguins.

Ces résultats analytiques sur la distribution du marqueur ne pouvant conduire qu'à des hypothèses, il est peut être plus intéressant de mesurer la durée totale de diffusion dans l'organisme ; d'après les résultats de nombreux auteurs (tabl. 4), cette durée varie de 30 à I $80 \mathrm{mn}$ chez les petits animaux (Rat, Souris, Chat, Chien, Lapin), chez l'Homme, le Singe et le Porc. Elle est plus longue chez les ruminants de 120 à $480 \mathrm{mn}$.

Ainsi, l'analyse qualitative de la distribution de l'eau marquée dans l'organisme nous montre qu'elle est complète dans toute l'eau corporelle, espaces extracellulaire et intracellulaire, et qu'elle se produit assez rapidement.

La diffusion semble en outre très homogène dans les différents compartiments hydriques étudiés par plusieurs auteurs : eau tissulaire du foie, du cerveau, des musclęs et des os, fluide intersticiel (salive, suc gastrique, contenu digestif, fluide amniotique et allantoïdique) et produits d'excrétion (sueur, urine, vapeur d'eau expirée), (EDELMAN, I952; HURsT et al., I952; THOMPSON r954; Pinson et Langham ,1957; Till et Downes, r962 ; Moens et al., 1963). Foot et GreenHALGH (1970) en particulier, ont mesuré la concentration du marqueur sur 6 brebis, dans dix compartiments hydriques différents : les différences moyennes observées sont inférieures à $3 \mathrm{p}$. roo de la concentration du marqueur dans le plasma après équilibre. Cette homogénéité n'est pas surprenante étant donné que l'eau marquée se comporte pratiquement comme l'eau ordinaire dans les phénomènes de diffusion, tout du moins dans la gamme de concentration utilisée lors de 1'application de cette méthode (Edelman, I952 ; Prentice, I952 ; Moens, I963). Ainsi, selon la formule de Foy et Schnieden (1960), l'eau marquée n'est pas « reconnue par le métabolisme ». 
TABLEAU 4

Caractéristiques de la diffusion de l'eau marquée dans l'organisme chez différentes espèces

\begin{tabular}{|c|c|c|c|c|}
\hline Auteurs & Animaux & Marqueurs & Teq (mn) & $T(j)$ \\
\hline 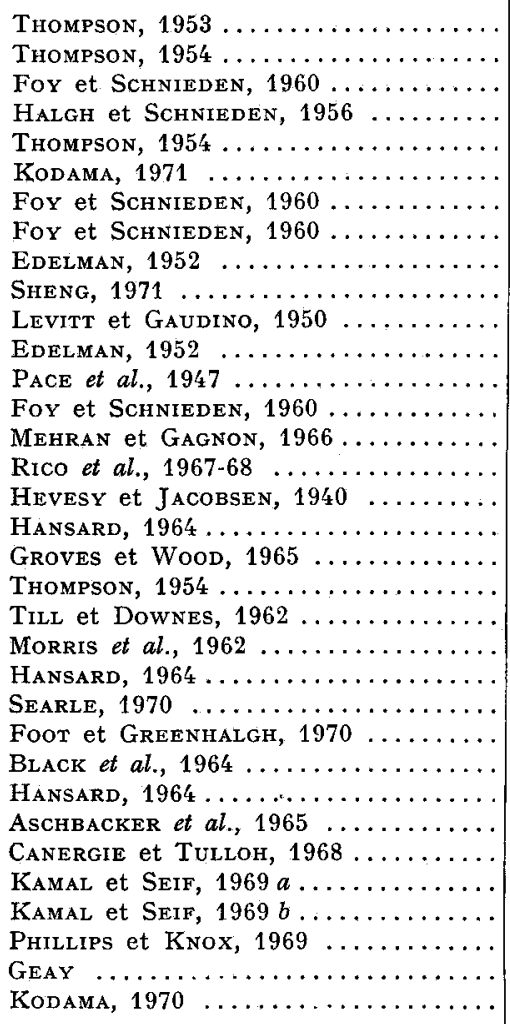 & $\begin{array}{l}\text { Rat } \\
- \\
\text { - } \\
\text { Souris } \\
\text { Hamster } \\
\text { Cobaye } \\
\text { Chat } \\
\text { Chien } \\
\text { - } \\
\text { Lapin } \\
\text { - } \\
\text { - } \\
\text { Porc } \\
\text { Ovins } \\
\text { - } \\
\text { - } \\
\text { - } \\
\text { Bovins } \\
\text { - } \\
\text { - } \\
\text { Singe } \\
\text { - }\end{array}$ & $\begin{array}{c}\mathrm{TOH} \\
- \\
\mathrm{D}_{2} \mathrm{O} \\
\mathrm{TOH} \\
\\
\mathrm{D}_{2} \mathrm{O} \\
\mathrm{TOH} \\
\mathrm{D}_{2} \mathrm{O} \\
\overline{\mathrm{TOH}} \\
- \\
- \\
- \\
\mathrm{D}_{2} \mathrm{O} \\
\mathrm{TOH} \\
\mathrm{D}_{2} \mathrm{O} \\
\mathrm{TOH} \\
- \\
- \\
- \\
- \\
\mathrm{D}_{2} \mathrm{O} \\
\mathrm{TOH} \\
- \\
- \\
- \\
- \\
- \\
\mathrm{TOH}\end{array}$ & $\begin{array}{c}60 \text { à } 300 \\
300 \\
360 \\
120 \text { à } 240 \\
360 \\
480 \\
300 \\
480 \\
360 \\
360 \\
480 \\
90\end{array}$ & 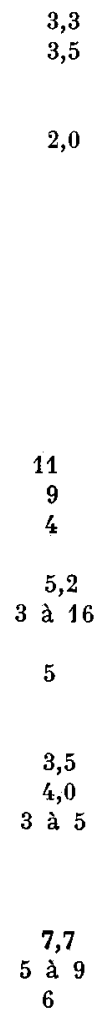 \\
\hline 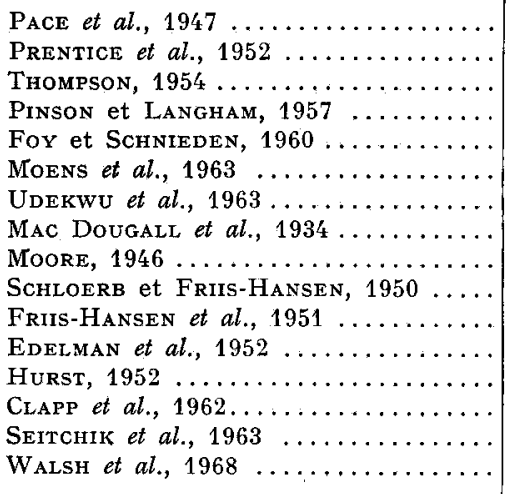 & $\begin{array}{l}\text { Homme } \\
- \\
- \\
- \\
- \\
- \\
- \\
- \\
- \\
- \\
-\end{array}$ & $\begin{array}{l}\mathrm{TOH} \\
- \\
- \\
- \\
- \\
\mathrm{D}_{2} \mathrm{O} \\
- \\
- \\
- \\
- \\
-\end{array}$ & $\begin{array}{c}60 \\
120 \\
80 \\
140 \\
120 \\
90 \text { à } 150 \\
90 \\
\\
60 \\
120 \\
150 \\
180 \\
120 \\
180 \\
600 \\
120\end{array}$ & $\begin{array}{c}10 \\
9 \\
11,5 \\
7,5 \\
2 \text { à } 19 \\
9 \\
9,5\end{array}$ \\
\hline $\begin{array}{l}\text { Chapman et Black, } 1967 \ldots \ldots \ldots \ldots \ldots \\
\text { Ouhayoun, } 1970 \ldots \ldots \ldots \ldots \ldots \ldots\end{array}$ & Poulet & $\stackrel{\mathrm{TOH}}{-}$ & $\begin{array}{l}120 \\
120\end{array}$ & $\overline{4} \overline{\mathrm{a}} 7$ \\
\hline
\end{tabular}

$\mathrm{T}_{\mathrm{eq}}=$ Temps nécessaire pour atteindre l'équilibre $(\mathrm{mn})$.

$\mathrm{T}=$ Demi-vie biologique du marqueur dans l'organisme (j). 
On observe ici une différence fondamentale entre l'eau marquée et l'antipyrine (HuRsT, 1952 DuMONT, 1958).

Rapide et homogène, la distribution du marqueur dans l'organisme semble aller au-delà de l'espace hydrique; en effet, il se produit des échanges d'hydrogène marqué avec des molécules organiques, des protéines surtout (MoEns, 1963). D'après les travaux de Smitr (1936) sur la Souris, la concentration en eau lourde dans l'eau de combustion des tissus, après injection d'une seule dose de marqueur, est égale à I $_{5}, 6$ p. Ioo de la concentration de l'eau marquée dans l'eau corporelle. La proportion passe à $\mathbf{~} 8,5 \mathrm{p}$. roo si on fait une infusion continue de marqueur. De plus, il apparaît que les échanges sont 3 à 4 fois plus importants dans la masse délipidée que dans les lipides. Ainsi, il semble que l'hydrogène marqué prenne tout d'abord la place d'hydrogène labile, puis celle d'hydrogène moins facilement échangeable. Ces résultats sont confirmés par ceux de Boxer (1944) et de Prentice (1952). Pinson (1952) a calculé ce que représentait quantitativement ce pool d'hydrogène de l'organisme : il serait de $0, \mathrm{I}$ p. roo du poids vif. Ce résultat est voisin de celui obtenu par Thомpson (1953). Expérimentalement, ce phénomène se traduit par une surestimation du volume hydrique corporel par l'espace de diffusion apparent de l'eau marquée. Cette erreur varie de 0,5 à 2 p. Ioo du poids vif chez l'homme (HEvesy et JACOBSEN, I940 ; Schloerb et Friss-Hansen, 1950 ; Prentice et al., 1952 ; Pinson, 1952) ; elle serait de 1 p. Ioo chez la souris (Thompson, I954), 4 p. Ioo chez le cobaye (Foy et Schnieden, I960), 5 p. Ioo chez le singe (Kodama, I970), 5 p. Ioo chez le porc (KAY et al., I966). Cette surestimation peut également être due en partie, à la rétention de l'hydrogène marqué de l'eau liée du muscle (HAzLEwood et al., 1969).

En conclusion, on peut résumer la distribution de l'eau marquée dans l'organisme par un schéma simplifié (fig. 2). L'eau marquée injectée diffuse en quelques minutes dans le sang et l'espace extracellulaire, tandis qu'elle pénètre plus lentement dans l'espace intracellulaire. La distribution est homogène dans les différents compartiments hydriques de l'organisme, mais une partie de l'hydrogène marqué peut être incorporée dans les molécules organiques.

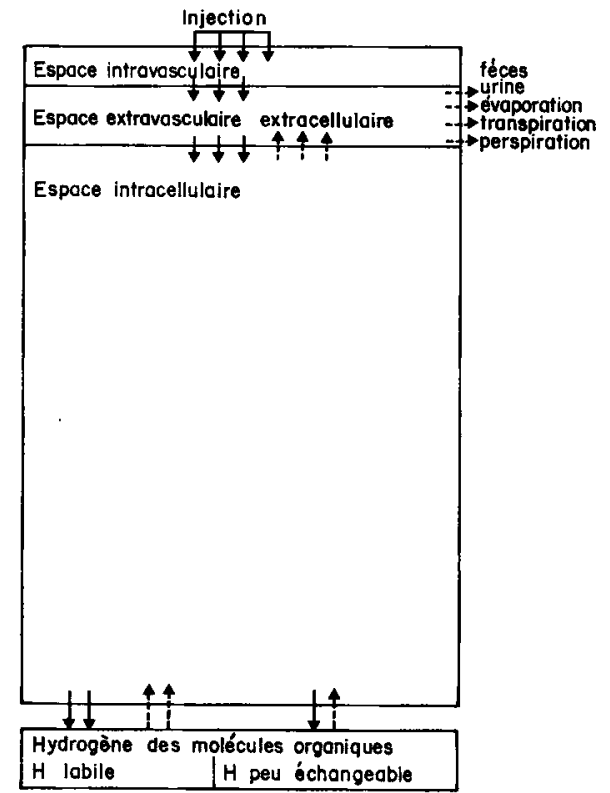

Fig. 2. - Schéma de la distribuiion de l'eau marquée dans l'organisme

Le nombre de flèches indique la rapidité des échanges,

- les flèches en trait plein correspondent à la dilution du marqueur,

- les flèches en pointillés correspondent à l'élimination du marqueur. 


\section{B. - Élimination du marqueur de l'organisme}

Bien qu'elle commence dès l'introduction de l'eau marquée, il n'est possible de l'étudier qu'après l'équilibre du marqueur dans l'eau corporelle, car avant ce stade, ses effets sont masqués par ceux de la dilution.

Nous avons vu que la concentration du marqueur est la même dans les produits d'excrétion (urine, sueur, eau expirée) que dans l'eau plasmatique. Par conséquent, l'organisme élimine les molécules d'eau marquée à la même vitesse que les molécules d'eau normale. L'élimination dépend donc en premier lieu du "turnover » de l'eau (BLACK et al., I964; BACKer et al., I965; McFarLANE et Howard, I966 ; SPRINGell, I968 ; PhILLIPS, 1969) et par conséquent des conditions atmosphériques ainsi que des quantités d'eau ingérées comme le montrent Till et Downes (1962) chez les bovins et surtout Pinson et Langham (1957) chez l'homme (fig. 3).

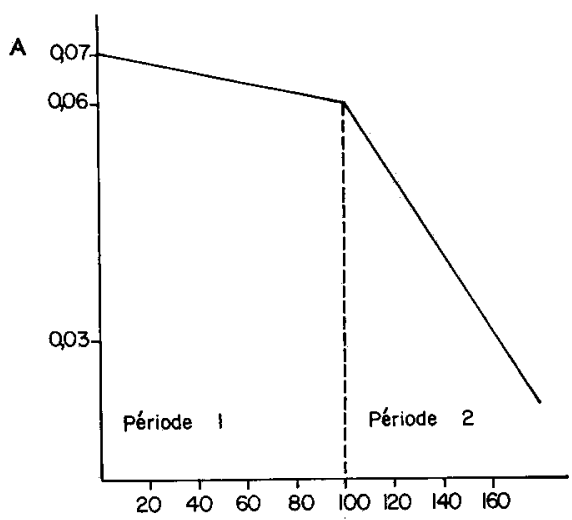

FIG. 3. - Influence de la quantité d'eau ingérée par jour sur la vitesse d'élimination du tritium de l'organisme (d'après Pinson et Langham, 1957)

$\mathrm{A}=$ Activité spécifique de l'eau plasmatique $(\mu \mathrm{Ci} / \mathrm{ml})$ Quantité d'eau ingérée par jour $\left\{\begin{array}{lll}\text { période } 1: 2,7 & \text { litres } \\ \text { période } 2: 12,8 \text { litres }\end{array}\right.$

Expérimentalement, on peut suivre la variation de la concentration du marqueur dans l'eau corporelle $\mathrm{C}$, ou de la quantité de marqueur $Q$ présente dans le volume hydrique $\mathrm{V}$ :

(r) $\frac{\mathrm{DQ}}{\mathrm{d} t}=\frac{\mathrm{dA}}{\mathrm{d} t}+\frac{\mathrm{dE}}{\mathrm{d} t}$

$\frac{\mathrm{dA}}{\mathrm{d} t}=$ Vitesse relative des apports du marqueur dans l'eau corporelle

$\frac{\mathrm{dE}}{\mathrm{d} t}=$ Vitesse d'élimination du marqueur de l'eau corporelle

(2) Comme $Q=\mathrm{CV}, \mathrm{dQ}=\mathrm{V} \mathrm{dC}$ car $\mathrm{V}$ est constant

(3) L'élimination est proportionnelle au " turnover " de l'eau ; soit $\alpha$ la quantité d'eau renouvelée par heure, soit : $\frac{\mathrm{dE}}{\mathrm{d} t}=-\alpha \mathrm{C}$

(4) Enfin, on peut admettre en première approximation, qu'après introduction du marqueur sous forme d'une seule injection et après diffusion complète dans l'organisme, les apports du marqueur dans l'eau corporelle sont nuls, soit $\frac{\mathrm{dA}}{\mathrm{d} t}=\mathrm{o}$ 
D'après ( 1$),(2),(3)$ et (4)

$$
\mathrm{V} \frac{\mathrm{dC}}{\mathrm{d} t}=-\alpha \mathrm{C}
$$

soit

$$
\frac{\mathrm{dC}}{\mathrm{C}}=-\frac{\alpha \mathrm{d} t}{\mathrm{~V}}
$$

En posant $\frac{\alpha}{\mathrm{V}}=k$ et en intégrant $\mathrm{C}=\mathrm{C}_{0} \mathrm{e}^{-k t}$, équation dans laquelle $\mathrm{C}_{0}$ est une constante qui représente la concentration théorique initiale du marqueur.

Cette forme théorique de l'évolution de la concentration plasmatique du marqueur après l'équilibre, correspond aux courbes que l'on peut obtenir expérimentalement (EDELMAN, 1952 ; Pinson et Langman, I957; Geay, résultats non publiés). Pour caractériser l'élimination du marqueur, on se sert surtout du paramètre $k$, vitesse horaire relative d'élimination, et de la demivie $\mathrm{T}$ du marqueur, temps auquel la concentration $\mathrm{C}$ est égale à $\frac{\mathrm{C}_{0}}{2}$.

$$
\frac{\mathrm{C}_{0}}{2}=\mathrm{C}_{0} \mathrm{e}^{-k T}
$$

soit : $-\log 2=-k \mathrm{~T}$

soit : $\mathrm{T}=\frac{\log 2}{k}$

D'après les résultats du tableau 4, T est variable suivant les espèces : 2 à 5 jours chez le Porc, 3 à 5 jours chez le Rat, le Bœuf et le Poulet, 6 à ro jours chez l'Homme; ces valeurs correspondent à un pourcentage de disparition horaire respectif de $1,15,0,73$ et 0,36 , ce qui est donc très faible.

En première approximation, la concentration plasmatique du marqueur ne dépend que de l'élimination et de la dose injectée, si l'on suppose que les apports ultérieurs de marqueur sont nuls : $\frac{\mathrm{dA}}{\mathrm{d} t}=\mathrm{o}$. En fait, ceci est partiellement faux, compte tenu de l'hydrogène marqué lié aux molécules organiques. En effet, cet hydrogène est restitué dans l'espace hydrique. THOMPSON (r953) a étudié ce phénomène sur le Rat, pendant une durée de $3^{\circ o}$ jours après l'injection d'eau tritiée (fig. 4). Il apparaît de ses résultats, que les différents tissus libèrent le tritium lié à des molécules organiques, à des vitesses différentes, et que, pour un tissu donné, il existe au moins deux compartiments correspondants à deux vitesses d'élimination différentes : le compartiment à élimination rapide correspond à l'hydrogène labile, l'autre à l'élimination plus lente et beaucoup plus étalée dans le temps, correspond à l'hydrogène plus fortement lié.

Le premier compartiment libère la majorité de l'hydrogène dans les premiers jours suivant l'équilibre, et les quantités libérées ne sont pas négligeables devant les quantités d'hydrogène marqué éliminées de l'organisme par l'eau. La variation de la quantité d'eau marquée présente dans l'espace hydrique corporel est alors égale à $\frac{\mathrm{dQ}}{\mathrm{d} t}=-\alpha \mathrm{C}+\frac{\mathrm{dA}}{\mathrm{d} t}$. Ainsi, la concentration du marqueur dans l'eau corporelle est supérieure à ce qu'elle serait sans cette restitution d'hydrogène marqué par les tissus.

Cette interprétation qui divise arbitrairement l'évolution de la concentration du marqueur dans l'eau en deux phases (diffusion et élimination) est schématique. Elle permet de séparer deux phénomènes simultanés. Fayet (197r) à la suite des travaux de Aubert et Milhaur (I960) ne fait pas cette distinction entre les deux phases, et par conséquent n'introduit pas la notion d'équilibre. FAYET représente l'évolution de la radioactivité spécifique du plasma $\left(R_{t}\right)$ par une équation de la forme $R_{t}=R_{1} e^{-\lambda 1 t}+R_{2} e^{-\lambda 2 t}$ dans laquelle $R_{1}, R_{2}$ sont les radioactivités spécifiques initiales des compartiments extracellulaires et intracellulaires. La sortie de l'eau de l'organisme est exprimée en vitesse tctale de sortie, plus utilisable que la période $T$ pour l'interprétation des résultats obtenus avec des animaux de taille comparable. La période T n'a l'intérêt que de per- 


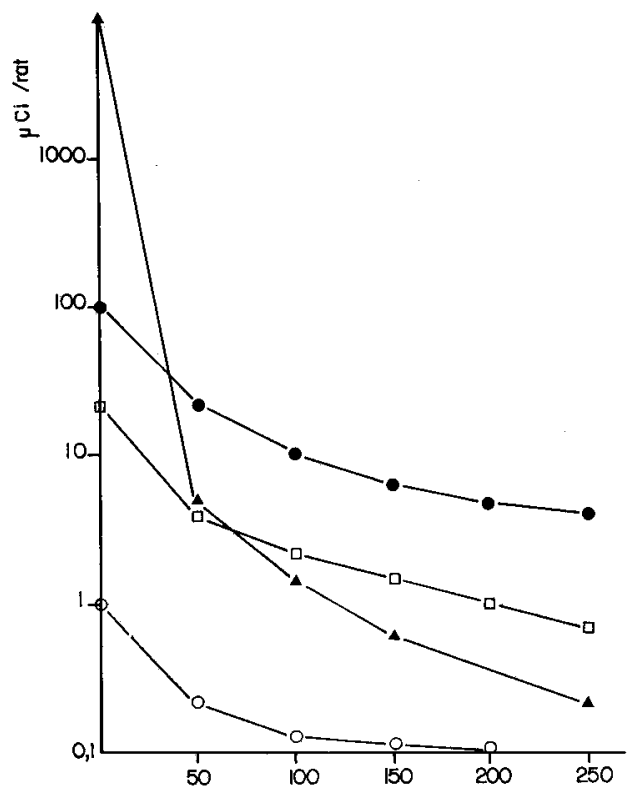

Fig. 4. - Cinetique de l'élimination du tritium de l'eau et de quelques tissus chez le Rat (d'après Thompson, I953)

- Activité de l'eau corporelle

- Activité du muscle

- Activité des dépôts adipeux

- Activité du cerveau

mettre d'établir une comparaison entre le métabolisme de l'eau d'animaux de taille très différente.

Quelle que soit l'interprétation retenue, les caractéristiques de la dilution de l'eau marquée dans l'organisme, semblent montrer que son espace de diffusion doit être un bon estimateur du volume hydrique corporel, moyennant peut-être quelques corrections, tenant compte de l'hydrogène marqué lié aux molécules organiques.

\section{III. - ESTIMATION PAR L'ESPACE DE DIFFUSION DE L'EAU MARQUÉE, DU VOLUME HYDRIQUE CORPOREL CHEZ L'ANIMAL, VIVANT}

Cette méthode à l'eau marquée a été très largement utilisée depuis les travaux de Hevesy et Hofer (1934). Dans la plupart des cas, et notamment en clinique humaine, on ne peut pas établir de comparaisons entre le volume hydrique estimé, et le volume réel (EDELMAN et al., I952 ; CeApP et al., I962; Morris et al., I962; Seitchik et al., I963; Panaretro, r964; Kamal et SEIF, I969 $a$ et $b$; OWEN et al., I969; GoRDON et al., I97 I ; Yates et al., r97I). Avant d'essayer d'estimer la précision de cette méthode, il est souhaitable de résumer le dispositif expérimental utilisé par les différents auteurs dont nous avons analysé les travaux.

Les animaux sont mis au jeûne partiel, ou total, pendant une durée qui peut varier de o à 48 heures avant l'injection du marqueur à 7 à Io heures après cette injection dans le cas des ruminants, c'est-à-dire jusqu'à la fin des prélèvements d'échantillons de sang. 
L'introduction de l'eau marquée se fait généralement par voie intraveineuse chez les animaux, car l'équilibre est atteint plus rapidement qu'après l'ingestion du marqueur (TILI et Downes, I962). Dans le cas de l'Homme, chez lequel cette méthode d'estimation de la composition corporelle a été énormément utilisée (Hevesy et Hofer, I934; Friss-Hansen et al., I95I; Walsh et al., r968), l'introduction du marqueur se fait dans la plupart des cas par voie orale, pour des raisons de simplicité.

Les doses utilisées sont assez variables suivant les auteurs : environ $2 \mathrm{mCi}$ par $100 \mathrm{~kg}$ de poids vif en ce qui concerne l'eau tritiée, $O, \mathbf{I}$ à I 1 par roo $\mathrm{kg}$ de poids vif en ce qui concerne l'eau lourde. Ces doses sont surtout liées à la sensibilité des méthodes de dosage du marqueur dans l'eau plasmatique (Foor et GreEnHalGh, I970).

L'estimation du volume hydrique a presque toujours été faite à partir de la concentration $\mathrm{du}$ marqueur après équilibre $\left(\mathrm{C}_{e q}\right)$ en admettant que celle-ci était voisine de la concentration initiale $\left(C_{0}\right)$ (Levitt et Gaudino, 1950; Reid et al., I958). Seuls Aschbacher et al. (I965), Rico et al. (1967-68), Krittle (1970), Kodama (197 ז) et Sheng et Huggins (1971) ont calculé le volume hydrique d'après la concentration initiale théorique du marqueur, obtenue par extrapolation.

La répétabilité sur un même animal de l'estimation du volume hydrique à l'aide de l'eau tritiée est bonne. TIll et Downes (r962) avec des ovins, observent des différences toujours dans le même sens et inférieures à $5 \mathrm{p}$. Ioo du poids vif entre les résultats obtenus à 7 heures d'intervalle, les animaux restant au jeûne complet pendant cette période. On peut estimer que la répétabilité est meilleure car la diminution du volume hydrique peut s'expliquer par le "turnover " de l'eau. Sheng et HugGins (1971) confirment ces résultats avec 43 chiens : ils trouvent une différence moyenne de $2,5 \mathrm{p}$. roo du poids vif sur deux mesures faites à un jour d'intervalle. WaLSH (I968) obtient des résultats identiques sur l'Homme avec l'eau lourde.

La précision de la méthode peut être mesurée en comparant l'espace de diffusion du marqueur à la quantité d'eau corporelle mesurée par dessiccation, cette méthode étant prise comme référence. D'après les résultats du tableau 5 , l'espace de diffusion de l'eau marquée surestime de I à I 5 p. Ioo la quantité d'eau corporelle mesurée par dessiccation, la moyenne étant voisine de 4 p. roo. L'antipyrine donne en moyenne une estimation plus proche de la valeur réelle (SoBERman et al., I949; HURST, I952; Farber et Soberman, 1956), mais la dispersion des résultats est beaucoup plus importante que celle obtenue avec l'eau marquée (Panaretro et Till, I963; Hansard, I964).

Bien que l'espace de diffusion de l'eau marquée soit supérieur au volume hydrique corporel (V), le coefficient de corrélation entre ces deux valeurs est élevé : 0,96 à 0,99 dans le cas de l'eau tritiée, un peu plus faible dans le cas de l'eau lourde $(0,93)$ (tabl. 4). Le coefficient de variation de la régression de $\mathrm{V}$ sur $\mathrm{E}$ varie de 2 à $7 \mathrm{p}$. roo du volume hydrique corporel avec l'eau tritiée, il est voisin de 4 p. roo dans le cas de l'eau lourde (tabl. 5).

Les sources de variation de ces résultats peuvent nous permettre d'envisager des améliorations éventuelles de la méthode. En ce qui concerne la surestimation du volume hydrique par l'espace de diffusion, elle est inévitable, compte tenu du passage d'une certaine quantité d'hydrogène marqué dans des molécules organiques (MoENs et al., 1963). On peut cependant la réduire, en estimant l'espace de diffusion à partir de la concentration initiale théorique (Co) du marqueur. En effet, lorsqu'on calcule $\mathrm{E}$ à partir de la concentration à l'équilibre $\left(\mathrm{C}_{\boldsymbol{e} q}\right)$, on néglige les pertes d'eau qui peuvent se produire pendant la diffusion du marqueur, c'est-à-dire pendant une durée voisine de 6 à 7 heures dans le cas des ruminants (Till et Downes, I962 ; Foot et Greenhalgh, 1970). Cette erreur représente chez les ovins, selon les travaux de Till et Downes (I962), 4 à 5 p. roo du volume hydrique. Elle ne serait chez les bovins que de I d 2 p. Ioo, selon les résultats de Canergie et Tulloh (1968). En fait, elle est fonction du "turnover " de l'eau de l'animal ; elle est donc variable avec les conditions du jeûne précédant la mesure comme le montrent les travaux de Panaretro (1963 et 1968) et de SEARLe (1970). Cette surestimation paraît plus élevée chez les ruminants que chez les monogastriques (tabl. 5). 
ESPACES DE DIFFUSION DE L'EAU MARQUÉE

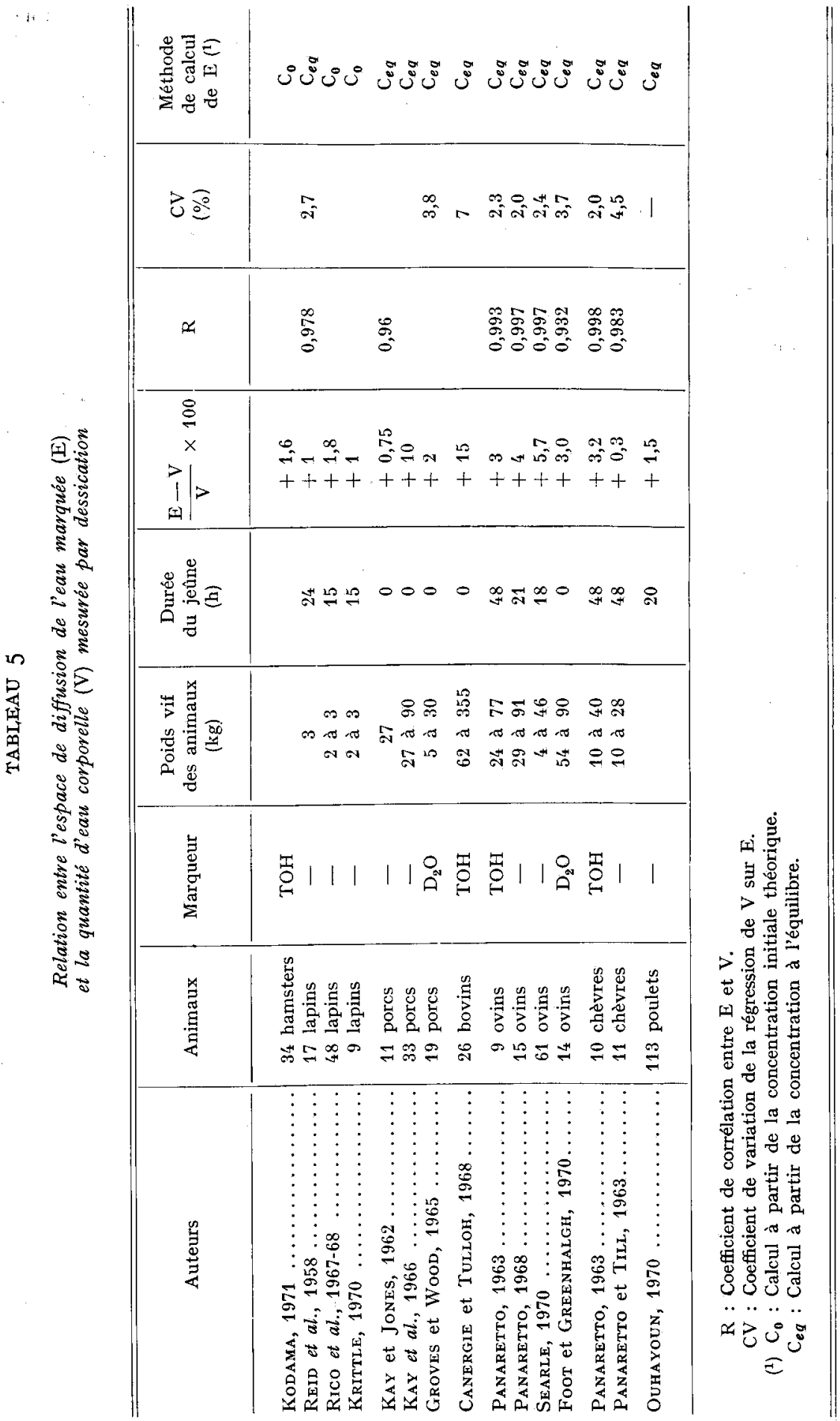


Ainsi, afin de réduire cette erreur, et surtout de la rendre plus reproductible, il semble préférable de calculer l'espace de diffusion du marqueur à partir de sa concentration initiale théorique dans le plasma (Rico et al., I967 ; Kodama, I97o ; Kritrle, I970).

Cependant, quelle que soit la précision avec laquelle on peut estimer la quantité d'eau contenue dans un animal, il se pose le problème de la signification de cette mesure : en effet, l'eau du contenu digestif représente une part importante de l'eau totale chez les monogastriques : 7 p. Ioo chez le Rat, I 5 p. Ioo chez le Lapin, 20 p. Ioo chez le Cobaye (CızeK, 1954). Ce pourcentage est plus élevé encore chez les ruminants : I 7 p. Ioo chez les ovins, 20 p. Ioo chez les caprins, $25 \mathrm{p}$. 10o chez les bovins après une durée de jeûne variable de I à 48 heures (Whiting et al., I960; Panaretto, 1963 ; Panaretro, 1968). Les variations sont relativement importantes d'un animal à un autre (3 p. Ioo du volume hydrique total) ; elles sont beaucoup plus faibles dans le temps sur un même animal alimenté avec la même quantité du même aliment. Enfin, la part de l'eau du contenu digestif dans l'eau totale semble diminuer légèrement avec le jeûne total (MAcFADDEN et Richards, I956 ; Panaretto, I968).

A défaut de méthode précise permettant d'estimer la quantité d'eau contenue dans le tube digestif (REID et al., I957 ; PANARetro et REID, I964), il semble donc nécessaire de normaliser les conditions de mesure afin d'avoir des résultats aussi comparables que possible. Dans ce but, la plupart des auteurs font précéder la mesure d'une durée de jeûne total ou partiel variant de I 5 à 48 heures, d'autres laissent les aliments et l'eau à la disposition des animaux jusqu'au moment de l'injection du marqueur, afin de ne pas perturber le schéma expérimental. Peut-être serait-i] souhaitable d'étudier de façon précise l'influence d'un jeûne de courte durée, sur la quantité d'eau contenue dans le tube digestif, d'une part, et d'estimer les répercussions de ce choc sur le gain de poids des animaux d'autre part.

En conclusion, il existe une liaison forte $(r>0,96)$ entre le volume hydrique corporel et l'espace de diffusion de l'eau marquée. L'erreur résiduelle de la régression varie de 2 à $7 \mathrm{p}$. Ioo du poids vif de l'animal, quelle que soit l'espèce considérée. On pourrait certainement améliorer

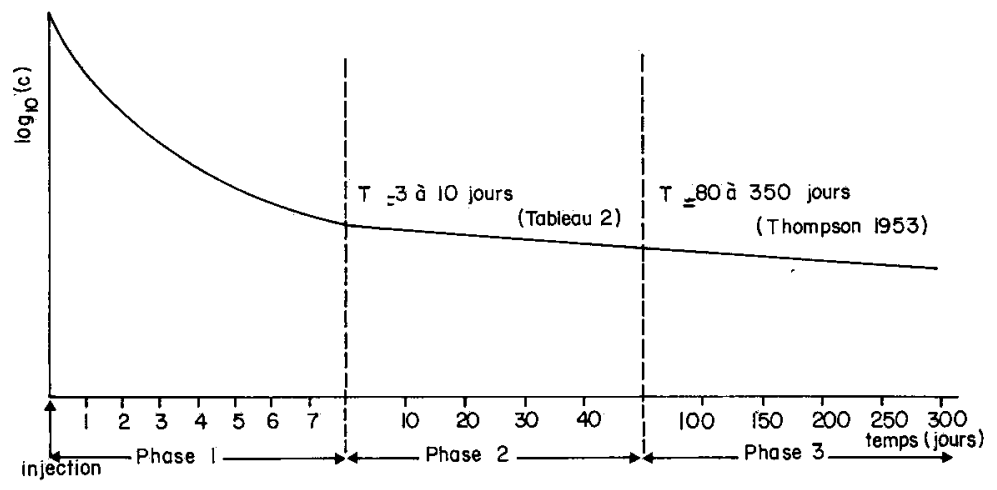

FIG. 5. - Évolution schématique en fonction du temps de la concentration plasmatique du tritium dans l'eau plasmatique

$c=$ concentration dans l'eau plasmatique de l'eau marquée

la précision de cette mesure en calculant le volume apparent de diffusion du marqueur à partir de la concentration théorique initiale obtenue par extrapolation de la droite d'élimination. Malgré sa précision, cette méthode d'estimation de l'eau corporelle se heurte à un écueil au niveau de la signification de cette mesure, compte tenu de l'importance relative, et des variations de la quantité d'eau contenue dans le tube digestif. 


\section{IV. - ESTIMATION DE LA TENEUR EN LIPIDES D'UN ANIMAL, A PARTIR DE LA MESURE DE L'ESPACE DE DIFFUSION DE L'EAU MARQUÉE}

Comme nous l'avons vu, il existe une liaison entre les teneurs en eau et en lipides du corps vide d'un animal, la lipogenèse entraînant une diminution de la teneur en eau (MURRay, I922; Moulton, I923 ; PaCe et Rathbun, I945; Reid et al., I968; Garrett et Hinman, 1969) ; il est donc normal que la teneur en lipides soit statistiquement liée à l'espace de diffusion d'un marqueur de l'eau, estimateur de l'eau corporelle totale.

D'après les résultats obtenus par plusieurs auteurs (tabl. 6), cette liaison est en effet relativement forte $(r=-0,93$ à $-0,99)$ dans le cas de l'eau tritiée. Elle est plus faible dans celui de l'eau

\section{TABIEAU 6}

Relation entre l'espace de diffusion de l'eau marquée (E) et le pourcentage de lipides corporels ( $\mathrm{L}$ p. Ioo)

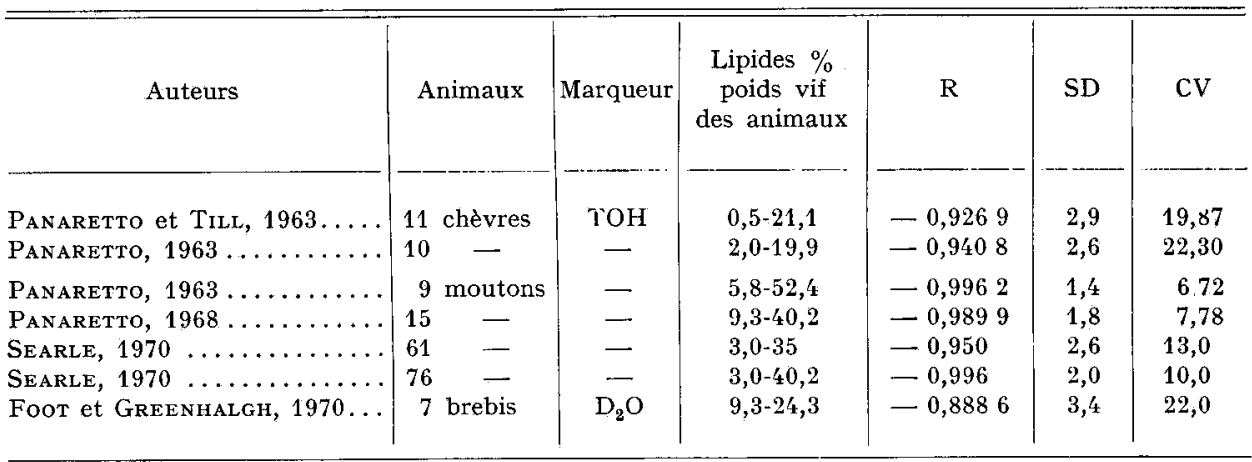

$\mathrm{R}=$ Coefficient de corrélation entre $\mathrm{E}$ et $\mathrm{L}$.

$\mathrm{SD}=$ Écart type résiduel de la régression de $\mathrm{L}$ sur $\mathrm{E}$.

$\mathrm{CV}=$ Coefficient de variation de la régression de $\mathrm{L}$ sur $\mathrm{E}$.

lourde $(-0,88)$ mais il s'agit de résultats relatifs à des brebis gestantes (Foor et GreENHALGH, 1970) animaux qui sont certainement en période de rétention hydrique importante (SEITchIK et al., 1963). Pour comparer les résultats entre eux, nous avons présenté sur la figure 6 les droites de régression calculées par différents auteurs. Les droites sont très voisines les unes des autres. Elles sont bien distinctes des courbes de PACE et Rathbun (1945) et Reid et al. (I955), représentant la teneur en lipides en fonction de la teneur en eau, toutes deux exprimées en pourcentage du poids vif vide. Cet écart paraît normal compte tenu de l'importance de l'eau du contenu digestif (8 à I6 p. Ioo du poids vif) et de la surestimation du volume hydrique par l'espace de diffusion de l'eau marquée.

La précision de l'estimation est meilleure dans le cas des ovins que dans celui des caprins (tabl, 6) ; l'écart-type résiduel de la régression varie de I,4 à 2,9 p. Ioo (en lipides p. Ioo du poids vif), ce qui correspond à un intervalle de con fiance variable de 2,8 à 5,8 p. roo dans le cas de l'eau 
tritiée. On ne peut pas tenir compte des résultats obtenus avec l'eau lourde sur les brebis en raison de leur état physiologique.

On peut certainement améliorer cette précision en mesurant de façon plus précise le volume hydrique de l'animal, mais surtout en essayant d'amoindrir l'influence du contenu digestif chez les ruminants. En effet, la relation qui existe entre la teneur en eau et la teneur en lipides est surtout valable au niveau du corps vide. Cette relation reste d'autant plus étroite au niveau du corps entier que le volume hydrique contenu dans le tractus digestif est comparable entre deux mesures.

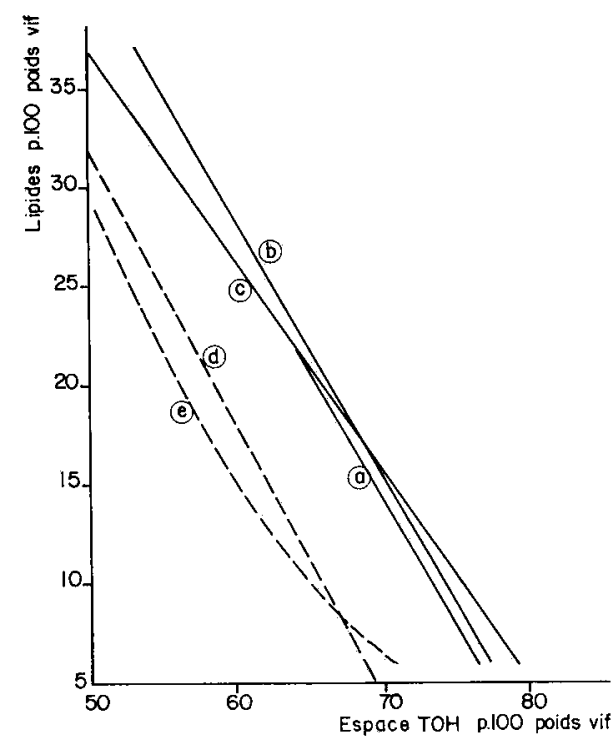

FIG. 6. - Droites de régression entre l'espace de diffusion de l'eau tritiće et les lipides corporels exprimés en pourcentage du poids vif

a) Panaretro et Till ( I $\left._{9} 63\right), 2$ i chevres.

b) Panaretto (I963), 9 moutons.

c) SeArle (i97o), 6i moutons.

d) Équation de PACE et al. (I945) : lipides p. Ioo poids vif vide/eau p. Ioo poids vif vide.

e) Équation de ReIn et al. ( 1955 ) : lipides p. roo poids vif vide/eau p. Ioo poids vif vide.

\section{CONCLUSION}

L'étude de la diffusion de l'eau marquée (eau lourde et eau tritiée) permet de penser qu'elle est un bon marqueur de l'eau; sa diffusion est rapide, de I à 7 heures suivant les espèces, elle est très homogène dans les différents compartiments hydriques de l'organisme. L'hydrogène marqué peut être incorporé de façon plus ou moins stable dans les molécules organiques mais on peut corriger cette erreur en diminuant le volume apparent occupé par le marqueur de 2 p. Ioo du poids vif. L'élimination du marqueur est lente, de o, I à $0,3 \mathrm{p}$. roo par heure de la quantité contenue dans l'organisme, ce qui permet de mesurer avec précision sa concentration dans l'eau corporelle après l'équilibre.

Dans la pratique, l'espace de diffusion de l'eau marquée reflète bien le volume hydrique total de l'animal : le coefficient de corrélation entre ces deux volumes est supérieur à 0,96 tandis que l'erreur résiduelle de la régression est inférieure à $2,5 \mathrm{p}$. xoo du poids vif. L'estimation de la teneur en lipides de l'organisme à partir de cette mesure est un peu moins précise : le coefficient de corrélation varie de $-0,92$ à $-0,99$, l'erreur résiduelle peut atteindre 2,9 p. roo (en lipides p. Ioo du poids vif). 
Cette méthode paraît d'autant plus prometteuse qu'on peut envisager deux possibilités d'améliorer sa précision. D'une part, on peut certainement estimer avec plus de précision le volume hydrique total de l'organisme, en calculant l'espace de diffusion à partir de la concentration initiale théorique du marqueur. D'autre part, il semble nécessaire d'approfondir les connaissances en ce qui concerne la composition du contenu digestif chez les ruminants, de façon à utiliser la méthode dans des conditions aussi comparables que possible en ce qui concerne la quantité d'eau contenue dans le tube digestif.

Cette méthode indirecte d'estimation de la composition corporelle, surtout utilisée en clinique humaine, rendrait d'énormes services dans des études sur animaux en état physiologique normal, si on pouvait adapter sa précision aux variations de composition corporelle relativement faibles qu'on désire mettre en évidence.

Enfin, le choix entre l'eau lourde et l'eau tritiée ne peut être fondé que sur des critères techniques car les résultats obtenus avec chacun de ces marqueurs sont assez comparables. Ainsi il faut tenir compte des problèmes de contamination par les éléments radioactifs des animaux destinés à la boucherie. Il faudrait apprécier également les techniques à mettre en œuvre et leur précision pour doser ces marqueurs dans l'eau (MoENs et al., I963; Graystone et al., 1967).

Rę̧u pour publication en novembre 1972.

\title{
SUMMARY
}

\author{
FSTIMATION OF ANIMAL, BODY COMPOSITION \\ BY LABEILIED WATER DIFFUSION SPACES. REVIEW
}

The method of measuring in vivo body composition, based on labelled water dilution, seems the most promising among the indirect methods.

According to numerous studies during the past $4^{\circ}$ years on this subject, it appears that labelled water (heavy or tritiated water) gives the best results.

Diffusion in body water is fast ( $\mathrm{I}-7$ hours, depending on the species), it is homogenous in the various water compartments of the organism, and label elimination is slow (I p. Ioo per hour of the dose contained in the organism).

The relationship between labelled water diffusion space and true hydric volume is strong $(r>0.96)$; it is weaker between this diffusion space in vivo and the amount of fatty matter in the animal $(r=-0.92$ to -0.99$)$.

The exactness of this method of measurement is limited, especially in ruminants, by the variability of the quantity of water in the digestive tract.

\section{RÉFÉRENCES BIBLIOGRAPHIQUES}

Aschbacher P. W., Kamal T. H., Cragle R. G., I965. Total body water estimation in dairy cattle using tritiated water. J. Anim. Sci., 24, 430-433.

Aubert J.-P., Milhaud G., I960. Méthodes de mesure des principales voies du métabolisme calcique chez l'Homme. Biochim. Biophys. Acta, 39, 122-139.

Backer N. F., Black A. L., ANand R. S., Fisk R. A., I965. Body water turnover in cattle with parasitic gastroenteritis. Exp. Parasitology, 17, $27 x-276$.

Black A. L., Backer N. F., Bartegy J. C., Chapman T. E., Phillips R. W., I964. Water turnover in cattle. Science, 144, 876-878.

Bowes H. J. M., rg6o. Biological fractionation of isotopes. Int. J. Appl. Rad. Isot., 7, 26I-266. 
Boxer J. E., Dewite-Stetren J., I944. Studies in carbohydrate metabolism : II. The glycogenic response to glucose and lactate in the previously fasted rat. J. Biol. Chem., 155, 237-242.

Brodie B. B., Berger E. Y., Axelrod J., Dunning M. F., Porosowska Y., Steele J. M., I95T. Use of N-Acetyl 4 Aminoantipyrine (NAAP) in measurement of total body water. Proc. Soc. Exp. Biol. Med., 77, 794-798.

Brozer J., Ig63. Body composition : part I. Ann. N. Y. Acad. Sci., 110, I-424.

Cachera R., Barbier P., r941. Cités par Dumont B. L., 1958. Méthodes indirectes de mesure de la graisse corporelle des mammifères. Ann. Nutr. Alim., 12, 95-I58.

Canergie A. B., Tulloh N. M., r968. The in vivo determination of body water space in cattle using the tritium dilution technique. Proc. Aust. Soc. Anim. Prod., 7, 308-3 3 .

Chapman T. E., Black A. L., I967. Water turnover in chickens. Poultry Sci., 46, 76I-765.

Cizek L. J., x954. Total water content of laboratory animals with special reference to volume of fluid within the lumen of the gastrointestinal tract. Amer. J. Physiol, 179, ro4-1 1o.

Clapp W. M., Butterfield L. J., O'Brien D., I962. Body water compartments in the premature infant, with special reference to the effects of the respiratory distress syndrome and of maternal diabetes and toxemia. Pediatrics, 30, 883-889.

Danowski T. S., r944. Cité par Dumont B. L., I958. Méthodes indirectes de mesure de la graisse corporelle des mammifères. Ann. Nutr. Alim., 12, 95-158.

Desmoulin B., 1970. La détermination de la densité corporelle. Journée de la Recherche porcine en France, 1970 .

Crown R. M., Damon R. A., I96o. The value of the I2th rib cut for measuring beef carcass yields and meat quality. J. Anim. Sci., 19, I09-II3.

Done J., Payne P. R., I957. The estimation of the dilution of tritium-labelled water of low activity in body fluids in man (Abst.). Proc. Nut. Soc., 16, VII-VIII.

Dumont B.-L., 1958. Méthodes indirectes de mesure de la graisse corporelle des mammifères. Ann. Nutr. Alim., 12, 95-158.

EDELMAN I. S., I952. Exchange of water between blood and tissues. Characteristics of deuterium oxide equilibration in body water. Am. J. Physiol., 171, 279-296.

Ederman I. S., Brooks L., Moore F. D., I952. Endogenous fat metabolism studied by heavy water dilution and nitrogen balance. J. Clin. Invest., 31, 626 .

FARber S. J., Soberman R. J., I956. Total body water and total exchangeable sodium in odematous states due to cardiac, renal or hepatic disease. J. Clin. Invest., 32, 779-787.

FAYET J. C., I97r. Plasma and faecal osmolality, water kinetics and body fluid compartments in neonatal calves with diarrhoea. Br. Vet. J., 127, 37-44.

Flexner L. B., Gellhorn A., Merrell M., 1942. Studies of rate of exchange of substances between blood and extravascular fluid : I. The exchange of water in the guinea pig. J. Biol. Chem., 144, 35-40.

Foot J. Z., Greenhalgh J. F. D., r97o. The use of deuterium oxide space to determine the amount of body fat in pregnant blackface ewes. Br. J. Nut., 24, 815-825.

Foy J. M., Schnieden H., rg6o. Estimation of total body water (virtual tritium space) in the rat, cat, rabbit, guinea pig and man, and the biological half life of tritium in man. $J . P h y s i o l ., 154,169-176$.

Friss-Hansen B. J., Holiday M., Stapleton T., Wallace W. M., i95I. Total body water in children. Pediatrics, 7, 321-327.

GarketT W. N., MEYer J. H., LofGreen G. P., 1959. The comparative energy requirements of sheep and cattle for maintenance and gain. J. Anim. Sci., 18, 528-536.

GarRetT W. N., HinmaN N., I969. Re-evaluation of the relationship between carcass density and body composition of beef steers. J. Anim. Sci., 28, I-5.

Geay Y., Beranger C., r969. Estimation de la composition de la carcasse de jeunes bovins à partir de la composition d'un morceau monocostal au niveau de la II ${ }^{\mathrm{e}}$ côte. Ann. Zootech., 18, 65-77.

Glascock R. F., Duncombe W. G., I954. Differential fractionation of hydrogen isotopes in liver and mammary gland. Biochem. J., 58, 440-447.

Gordon A. J., Topps J. H., BegG T. W., r97x. Total body water of rats as measured with different amounts of injected tritiated water. Proc. Nut. Soc., 30, $54 \mathrm{~A}$.

Graystone J., Seitchik J., Milch R., Shulman G. P., Cheek D. B., I967. Measurement of $\mathrm{D}_{2} \mathrm{O}$ in plasma water by freezing point elevation, falling drop, infra-red absorption, and gas chromatography. J. Lab. Clin. Med., 69, 885-892.

Groves T. D. D., Wood A. J., I965. Body composition studies on the suckling pig: II. The in vivo determination of total body water. Can. J. Anim. Sci., 45, I4-2I.

Haigh C. P., Schnieden H., r956. Virtual deuterium oxide space (total body water) in normal and protein deficient rats. J. Physiol., 131, 377-382.

Hamburger J., Mathe G., I952. Cités par Dumont B. L., r958. Méthodes indirectes de mesure de la graisse corporelle des mammifères. Ann. Nutr. Alim., 12, 95-I58.

Hankins O. G., Howe P. E., 1946. Estimation of the composition of beef carcass and cuts. Tech. Bull. U. S. Dept. Agric., $\mathbf{9 2 8 .}$

HANSARD S. L., r964. Total body water in farm animals. Am. J. Physiol., 206, 1369-1372. 
Hastings A. B., 1940. Cité par Kraybill H. F., Hankins O. G., BitTer H. L., I95I. Body composition of cattle : I. Estimation of body fat from measurement in vivo of body water by use of antipyrine. J. Appl. Physiol., 8, 681-689.

Haxhe J. J., 1964. Mesures des compartiments corporels. Méthodes et résultats. J. Physiol. (Paris), 56, 7-ro9.

Hazlewood C. F., Nichols B. L., Chamberlain N. F., Ig6g. Evidence for the existence of a minimum of two phases of ordered water in skeletal muscle. Nature, 222, 747-750.

Hevesy G., Hofer E., r934. Elimination of water from the human body. Nature, $134,879$.

Hevesy G., JAcoBSEN C. F., I940. Rate of passage of water through capillary and cell walls. Acta Phys. Scandinav., 1, I I-18.

Hopper T. H., I944. Methods of estimating the physical and chemical composition of cattle. $J$. Agric. Res., 68, 239.

HURST W. N., I952. Simultaneous determination of total body water by antipyrine and deuterium oxide : evaluation of the methods on oedematous subjects. J. Lab. Clin. Med., 39, 36-4o.

Hurst W. N., Schem F. R., Vogel W. C., 1952. Urine blood ratio of deuterium oxide in man. J. Lab. Clin. Med., 39, 4r-43.

Kamal T. H., Seif S. M., I $969 a$. Effect of natural and controlled climates of the Sahara, on virtual tritium space in Friesans and water Buffaloe. J. Dairy Sci., 52, I657-1663.

Kamal T. H., Seif S. M., I $969 b$. Changes in total body water and dry body weight with age and body weight in Friesians and water Buffaloes. J. Dairy Sci., 52, 1650-1656.

KAY M., Jones A. S., I962. The relationship between body density, body fat, and body water in living pigs (Abstr.). Anim. Prod., 4, 296.

Kay M., Jones A. S., SmarT R., I966. The use of tritiated water, 4-Aminoantipyrine and N. Acetyl4-Aminoantipiryne for the measurement of body water in living pigs. $B r . J$. Nutr., 20, 439-448.

Kodama A. M., 1970. Total body water of the pig-tailed monkey, Macaca nemestrina. J. Appl. Physiol., 29, 260-262.

KODAMA A. M., I97r. In vivo and in vitro determinations of body fat and body water in the hamster J. Appl. Physiol., 81, 218-222.

Kraybill H. F., Bitter H. L., Hankins O. G., r952. Body composition of cattle : II. Determination of fat and water content from measurement of body specific gravity. $J$. Appl. Physiol., 4, 575-583.

Kritrle J. P., 1970. Dosage de l'eau totale chez le Lapin par la méthode à l'oxyde de tritium. Thèse vêtérinaire. Toulouse.

LEDGer H. P., Hutchison H. F., I962. The value of the tenth rib as a sample joint for the estimation of lean, fat, and bone in carcass of East African zebu cattle. J. Agric. Sci., 58, 8I-88.

Levits M. F., Gaudino M., I950. Measurement of body water compartments. Am. J. Med., 9, 208-2I5.

Mac Dougall E. J., Verzar F., Erlenmeyer H., Gaertner H., I934. Heavy water in the animal body. Nature, 134, roo6-Ioo7.

MAC FADDEN D. L., RichardS C. R., I956. The determination of body composition by the use of antipyrine in aureomycin-fed dairy calves. J. Dairy Sci., 39, I439.

Mac Farlane W. V., Howard B., I966. Water content and turnover of identical twin Bos indicus. and Bos taurus in Kenya. J. Agric. Sci., 66, 297-302.

Martin J., Torreele G., I967. L'appréciation de la qualité des carcasses bovines par la découpe du morceau tricostal 7, 8, 9. Ann.. Zootech., 11, 217-224.

Mehran A. R., Gagnon A., Ig66. Mesure de l'eau échangeable chez le Lapin par une méthode rapide Arch. Int. Physiol. Bioch., 74, 549.

Moens R. S., Busset R., Collet R. A., Nagant de Deuxchaisnes C., Mach R. S., ig63. Détermination de l'eau totale par la méthode à l'eau tritiée. Schweiz. Med. Wschr., 93, 965-969.

Moore F. D., 1946. Datermination of total body water and solids with isotopes. Science, 104, 157-160.

Morris R. J. H., Howard B., Mac Farlane W. V., rg62. Interaction of nutrition and air temperature with water metabolism of merino wathers shorn in winter. A ust. J. Agric. Res., 13, 320-334.

Moulton C. R., Trowbridge P. F., Haigh L. D., 1922. Cités par Reid J. T., Wellington G. H., DunN H. O., r955. Some relationships among the major chemical components of the bovine body and their application to nutritional investigations. $J$. Dairy Sci., 38, I344-I359.

Moulton C. R., 1923. Cité par Kraybill H. F., Hankins O. G., Bitter H. L., i95i. Body composition of cattle : I. Estimation of body fat from measurement in vivo of body water by use of antipyrine. J. Appl. Physiol., 3, 68г-68g.

Murray J. A., r922. Cité par Kraybill H. F., Hankins O. G., Bitter H. L., i95i. Body composition of cattle : I. Estimation of body fat from measurement in vivo of body water by use of antipyrine. J. Appl. Physiol., 3, 68г-689.

Ouhayoun J., 1970. Estimation des lipides corporels du poulet par la méthode de dilution isotopique de l'eau tritiée. Ann. Biol. anim. Bioch. Biophys., 10, 621-630.

OWEN G., KERRY R. J., Davies H. M., I969. Total body water and weight changes in normal adults. Nature, 221, 47I-472. 
Pace N., Rathbun E. N., 1945. Studies on body composition : III. The body water and chemicaly combined nitrogen content in relation to fat content. J. Biol. Chem., 158, 685-691.

Pace N., Kline L., Schachman H. K., Harfenist M., I947. Studies on body composition: IV. Use of radioactive hydrogen for measurement in vivo of total body water. J. Biol. Chem., 168, 459-469.

Painter E. E., I940. Cité par Dumont B. L., I958. Méthodes indirectes de mesure de la graisse corporelle des mammifères. Ann. Nutr., Alim., 12, 95-158.

Panaretro B. A., I963. Body composition in vivo : III. The composition of living ruminants and its relation to the tritiated water spaces. Austr. J. Agric. Res., 14, 944-952.

Panaretro B. A., Till A. R., I963. Body composition in vivo : II. The composition of mature goats and its relationship to the antipyrine, tritiated water, N Acetyl-4-Aminoantipyrine spaces. $A$ ust. $J$. A gric. Res., 14, 926-943.

Panaretto B. A., Reid J. T,, r964. Body composition in vivo : V. The use of antipyrine and N-acetyl4-Amino-antipyrine for the estimation of gut water in living ruminants. Aust. J. Agric. Res., 15, 195-199.

Panaretro B. A., I968. Body composition in vivo: IX. The relation of body composition to the tritiated water spaces of ewes and wethers fasted for short periods. Aust. J.A gric. Res., 19, $267-272$.

PANARETio B. A., I968. Estimation of body composition by the dilution of hydrogene isotopes. In Body composition of animal and man, p. 200. Nal. Acad. Sci. Washington. Publ. $\mathrm{n}^{\circ}$ I598.

Phillips R. W., KNox K. L., 1969. Water kinetics in enteric disease of neonatal calves. J. Dairy Sci., 52, I664-I668.

Pinson E. A., I952. Water exchanges and barriers as studied by the use of hydrogen isotopes. Physiol. Rev., 32, 123.

Pinson E. A., Langham W. H., 1957. Physiology and toxicology of tritium in man. J. Appl. physiol., 10, 108-1 26.

Prentice T. C., Siri W., Berlin N. I., Hyde G. M., Parsons R. J., Joiner E. E., Lawrence J, H., 1952. Studies of total body water with tritium. J. Clin. Invest., 31, 412-418.

Rathbun E. N., Pace, N., 1945. Studies on body composition : I. Determination of total body fat by means of the body specific gravity. J. Biol. Chem., 158, 667-676.

Rico A. G., Lorgue G., Kritrue J. P., ig67. Détermination de l'eau totale chez le Lapin par la méthode de l'eau tritiée. Rev. Med. Vet., 118, 949-959.

Rico A. G., Lorgue G., I968. Détermination de l'eau totale chez le Lapin par la méthode de l'eau tritiée. Note II. Rev. Med. Vet., 119, 667-67I.

Reid J. T., Wellington G. H., Dunn H. O., I955. Some relationships among the major chemical components of the bovine body and their application to nutritional investigations. J. Dairy Sci., 38, I344-ז359.

REID J. T., BALCh C. C., HEAD M. J., 1957. Use of antipyrene and N-Acetyl-4-Aminopyrene in the measurement of body water and the intraluminal water of the gastro-intestinal tract of living cattle. Nature, 179, ro34.

ReID J. T., BALCH C. C., Glascock R. F., I958. The use of tritium, of antipyrene and of N-Acetyl4-Aminoantipyrene in the measurement of body water in living rabbits. Brit. J. Nutr., 12, 43-51.

Reid J. T., Bensadoun A., Bull L. S., Burton J. H., Gleeson P. A., Han I. K., Joo Y. D., Johnson D. E., Macmanus W. R., Paladines O. L., Stroud J. W., Tyrrell M. F., Van NieKERK B. D. H., Welinington G. H., Wood J. D., 1968. Changes in body composition and meat characteristics, accompanying growth of animals. Proc. Cornell. Nutr. Conf. Feed. Manuf., 1968, I8-37.

Schloerb P. R., Fris-hansen B. J., x950. The measurement of total body water in the human subject by deuterium oxide dilution. 'J. Clin. Invest., 29, 1296.

SEARLE T. W., I97o. Body composition in lambs and young sheep and its prediction in vivo from tritiated water space and body weight. J. A gric. Sci., 74, 357-362.

Seitchik J., Alper C., Szutka A., I963. Changes in body composition during pregnancy. Ann. N. Y. Acad. Sci., 110, 82 I-829.

Sheng H. P., Huggins R. A., I97I. Direct and indirect measurement of total body water in the growing Beagle. Proc. Soc. Exp. Biol. Med., 137, ro93.

Siri W. E., I956. The gross composition of the body. Adv. Biol. Med. Phys., 4, 239-280.

Smith P. K., Trace J., Barbour H. G., r936. The fate of deuterium in mammalian body. J. Biol. Chem., 116, 37 I-376.

Soberman R., Brodie B. B., Levy B. B., Axelrod J., Hollander V., Steele J. M., I949. The use of antyiprine in the measurement of total body water in man. J. Biol. Chem., 179, 3 I-42. $^{2}$

SPRingeld P. H., 1968. Water content and water turnover in beef cattle. Aust. J. Agric. Res., 19, I28-144.

Thompson R. C., 1953. Studies of metabolic turnover with tritium as a tracer: II. Gross study on the rat. J. Biol. Chem., 200, $731-743$.

Thompson R. C., I954. Biological applications of tritium. Nucleonics, 12, 3I-35.

Till A. R., Downes A. M., I962. The measurement of total body water in the sheep. Aust. J. Agric. Res., 13, 335-342. 
UDEkwu F. A. O., Kozoll D. D., MEYer K. A., I963. Determination of total body water with tritium oxide $\left(\mathrm{H}_{23} \mathrm{O}\right)$. J. Nuclear Med., 4, 6o-69.

Walsh G. C., Carruthers B. M., Seraglia M., Oslo M., rg68. Determination of total body water by deuterium oxide dilution and cryoscopy. J. Lab. Clin. Med., 72, 836-84r.

Whiting F., Balch C. C., CAmpung R. C., I96o. Some problems in the use of antipyrine and N-acetyl4-Amino-antipyrine in the determination of body water in cattle. Br. J. Nutr., 14, 5r9-533.

Yates N. G., MacFarlanne W. V., Ellis R., I97I. The estimation of milk intake and growth of beef calves in the field by using tritiated water. Austr. J. Agric. Res., 22, 29I-306. 